Spin Physics (SPIN2014)

International Journal of Modern Physics: Conference Series

Vol. 40 (2016) 1660084 (6 pages)

(C) The Author(s)

DOI: $10.1142 /$ S2010194516600843

\title{
Polarimetry for Stored Polarized Hadron Beams
}

\author{
E. J. Stephenson \\ Indiana University Center for Spacetime Symmetries \\ Bloomington, IN 47405 USA \\ stephene@indiana.edu
}

Published 29 February 2016

\begin{abstract}
Feasibility studies using $0.97-\mathrm{GeV} / \mathrm{c}$ polarized deuterons to determine the suitability of storage rings in the search for intrinsic electric dipole moments have led to new developments in storage ring polarimeters. Efficiency has increased with the use of thick carbon targets. The calibration of the effects of geometric and rate-dependent errors has made possible the subtraction of these systematic errors, resulting in sensitivities at or below $10^{-5}$ to polarization changes during the time of the store. Tagging polarimeter events with the clock time had made available the turn number for each event, making possible longitudinal profile and horizontal polarization measurements. These have been used to explore the correction of second-order contributions to decoherence using sextupole fields, yielding horizontal polarization lifetimes as long as 20 minutes. Such studies may be extended to protons and ${ }^{3} \mathrm{He}$.
\end{abstract}

Keywords: Polarimeter; storage ring; deuteron.

PACS numbers: 29.20.db, 29.27.Bd, 29.27.Hj, 29.85.Ca

\section{Introduction}

The idea of using a storage ring to contain a charged particle beam while it is monitored for evidence of an intrinsic electric dipole moment (EDM) in the radial electric field present in the particle frame was described in Ref. 1. This led to work at the COoler SYnchrotron $\left(\mathrm{COSY}^{2}\right)$ located at the Forschungszentrum Jülich with a polarized deuteron beam to demonstrate the feasibility of observing polarization rotations as small as $1 \mu \mathrm{rad}$ while the naturally-unstable component of the polarization along the beam velocity is preserved for times up to $1000 \mathrm{~s}$ using sextupole fields. The requirement for a polarimeter based on deuteron-carbon elastic scattering was met by the bar and ring scintillators of the EDDA detector, ${ }^{3-4}$ which led to an operating momentum of $0.97 \mathrm{GeV} / \mathrm{c}$ to make forward-angle deuteroncarbon elastic scattering events clearly visible. The beam was bunched to cancel the

This is an Open Access article published by World Scientific Publishing Company. It is distributed under the terms of the Creative Commons Attribution 3.0 (CC-BY) License. Further distribution of this work is permitted, provided the original work is properly cited. 


\section{E. J. Stephenson}

first-order decoherence from momentum spread and electron-cooled at the top of the acceleration ramp to minimize further decoherence from beta and synchrotron oscillations.

\section{Improved Polarimeter Efficiency}

The requirement to observe as many deuterons as possible from the beam to measure the polarization continuously led to using a thick $(17 \mathrm{~mm})$ carbon block located adjacent to the beam as the polarimeter target. Particle are brought to the target by (a) ramping a steering bump or (b) applying white electric field noise at a harmonic of the betatron frequency.

Extraction is a two-step process. First, the particle scatters from the inner face of the block, which induces a large betatron oscillation. On a subsequent turn, this particle enters the target front face. A typical distance from the edge is $0.2 \mathrm{~mm} .^{5}$

The measured efficiency for a minimum scattering angle of $9.2^{\circ}$ is $7 \times 10^{-4}$. This is consistent with an efficiency of $1 \%$ in an EDM polarimeter using a thicker target and a smaller inner scattering angle. The EDDA detector system provided an event set with an analyzing power of $A_{y}=0.45 \pm 0.03$.

\section{Subtraction of Polarimeter Systematic Errors for Vertical Polarization}

Systematic polarimeter errors are often managed by collecting data for opposite polarization states (spin up and down) and simultaneously on opposite sides of the beam (left and right). These four count rates are combined ${ }^{6}$ to produce an asymmetry:

$$
\epsilon=\frac{3}{2} p_{y} A_{y}=\frac{r-1}{r+1}, \quad r=\sqrt{\frac{C_{L+} C_{R-}}{C_{L-} C_{R+}}}
$$

The coordinate system has $z$ along the beam direction, $y$ vertical, and $x$ radially outward from the ring. This combination of count rates removes most first-order contributions to the asymmetry. At second-order, the remaining terms are those that depend on the rate of change of the analyzing powers with angle. These higherorder terms may be subtracted in general with the use of additional corrections:

$$
\epsilon=\frac{r-1}{r+1}+\left(\frac{\partial \epsilon}{\partial \phi}\right) \Delta \phi+\left(\frac{\partial \epsilon}{\partial W}\right) \Delta W
$$

where

$$
\phi=\frac{s-1}{s+1}, \quad s=\sqrt{\frac{C_{L+} C_{L-}}{C_{R+} C_{R-}}} \quad \text { and } \quad \mathrm{W}=\mathrm{C}_{\mathrm{L}+}+\mathrm{C}_{\mathrm{R}+}+\mathrm{C}_{\mathrm{L}-}+\mathrm{C}_{\mathrm{R}-}
$$

The partial derivatives in Eq. 2 are obtained in a calibration experiment in which large position and angle changes are made in the geometry and their effects measured for any polarization observables of interest. Similarly, rate effects $(W)$ are 


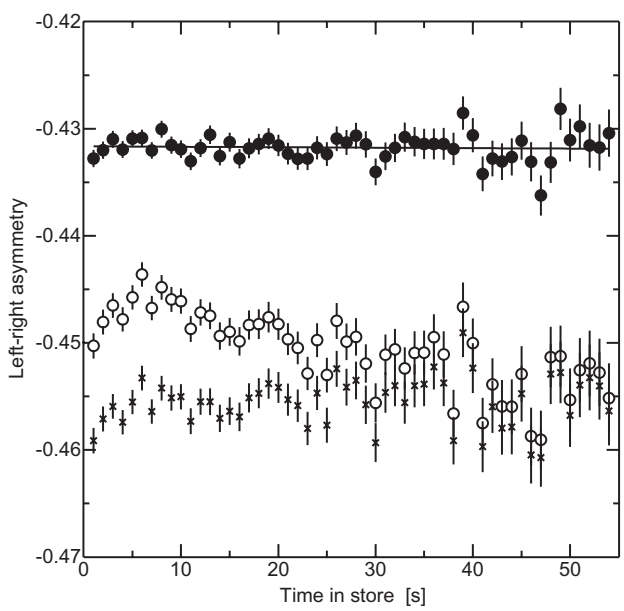

Fig. 1. Raw measurements (o) of the left-right asymmetry as a function of time of a constant vertical beam polarization. Application of the rate correction gives the crosses, and an additional application of the geometry correction gives the solid points. The straight line fit has a slope of $[-4 \pm 11] \times 10^{-6} \mathrm{~s}^{-1}$.

changes observed for different beam currents, noted here as the sum of the rates. In forward-angle scattering, position and angle changes may be related through the use of an effective distance to the detector, thus allowing $\phi$ to serve as the driving term for all geometric effects.

Figure 1 shows how raw data (circles) responds to rate (crosses) and geometric (dots) corrections, resulting is a polarization measurement that is flat with time at the level of $10^{-5}$. The precision of this correction is limited by counting statistics; there is no clear limit here on the application of the method at more sensitive levels.

\section{Horizontal Polarization Measurements}

Since the polarization rotates rapidly in the storage ring plane, a timing system was created to determine when the polarization is sideways so that polarimeter events could be selected and used for a measurement. For deuterons, the rotation frequency is $120 \mathrm{kHz}$. Each event was marked with the clock time which was calibrated against the signal from the bunching RF cavity. This provided a real turn number for every event. The fractional part of this number was used to locate the scattered particle relative to the center of the beam bunch, as shown in Fig. 2. The integer part, when multiplied by the spin tune $\left(\nu_{S}=G \gamma\right.$, where $G$ is the anomalous magnetic moment and $\gamma$ is the relativistic factor) gives the total angle through which the polarization has rotated since the start of data acquisition. The fractional part of this angle was used to bin polarimeter events according to their direction in the horizontal plane. Asymmetries calculated for these direction bins should vary sinusoidially. The spin tune value was searched over a narrow range in order to maximize the amplitude of the sine wave. The resulting magnitude was assigned to the horizontal polarization. 


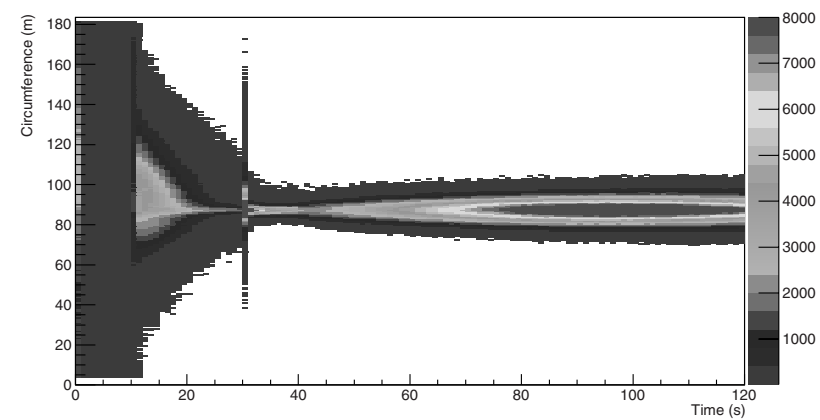

Fig. 2. The distribution of events around the COSY ring circumference as a function of time in the store. Bins with fewer than 5 events have been set to zero for clarity. Electron cooling with bunching reduces the width. Other features represent parts of the extraction process onto the carbon target.

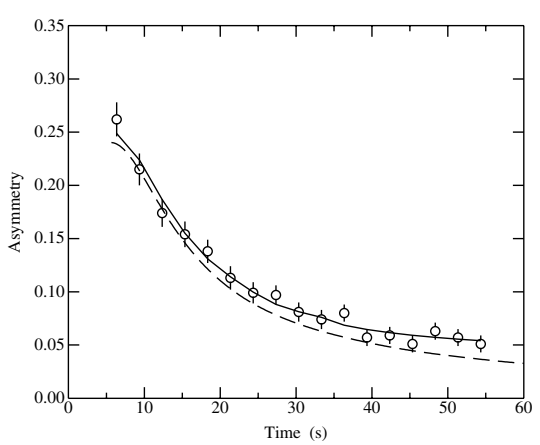

Fig. 3. Values of the down-up asymmetry magnitude as a function of time during the store. The dashed curve is a template for the time dependence based on the decoherence from betatron oscillations. A correction for a magnitude estimate yields the solid curve.

An example of such data is shown in Fig. 3. The polarization falls with time due to decoherence from betatron oscillations. A model of such a decline is shown by the dashed curve. The search for the largest polarization magnitude in each time bin leads to an overestimate. When this effect is included, the solid curve is the result (a good match with these data). A polarization lifetime may be assigned to these data as the time required to reach $61 \%$ of the original value (in analogy with the Gaussian width).

\section{Lengthening the Polarization Lifetime}

Betatron oscillations lead to polarization decoherence (bunched beam) because the path length around the ring, and consequently the particle speed and spin tune, increases. Such an effect may be compensated by introducing additional sextupole fields into the ring. These fields also change the chromaticity, and it is expected ${ }^{7-8}$ 
that the best polarization lifetime occurs when the chromaticity vanishes. Changes to the chromaticity are linear in the sextupole field strength (Section 2.1.1 of Ref. 9), so it is expected that the reciprocal of the polarization lifetime varies linearly with the additional sextupole strength, as shown in Fig. 4. The slope of the data was increased by applying electric white noise to the beam in the horizontal direction, thus increasing the size of the betatron amplitude distribution. The place where the straight line crosses zero in the sextupole strength needed to produce the longest polarization lifetime.

Recently a study was completed making a thorough exploration of the relationship between chromaticity and polarization lifetime. There are three sextupole

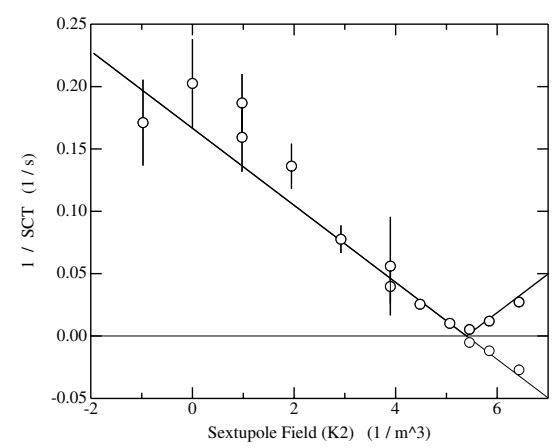

Fig. 4. Measurements of the reciprocal of the horizontal polarization lifetime as a function of the strength of the magnets in the COSY MXS sextupole family. The black line is the absolute value of a straight line. A continuation above the zero is shown in gray with the sign of the data points reversed.

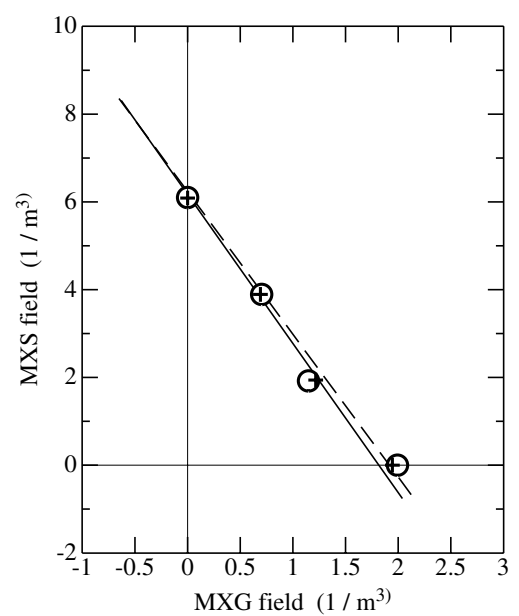

Fig. 5. Preliminary demonstration of the coincident positions of best polarization lifetime and zero chromaticity in a 2-D space of sextupole currents for the MXS (large $\beta_{X}$ ) and MXG (large D) magnet families. Further discussion is contained in the text. 
families: MXS (large $\beta_{X}$ ), MXL (large $\beta_{Y}$ ), and MXG (large dispersion). The values of the X and Y chromaticity lie in a plane as a function of MXS and MXG. The intersection of these planes with zero chromaticity is a line in the MXS by MXG plane (see Fig. 5) shown as solid or dashed respectively. By choosing the magnet strength to be MXL $=-0.2 \mathrm{~m}^{-3}$, it was possible to make these lines nearly coincident. These errors from the chromaticity tune measurements are \pm 0.3 in MXS and \pm 0.1 in MXG, so these lines are consistent with each other.

Horizontal polarization measurements were made for two COSY setups: (a) a heated beam having a large horizontal size (large $\mathrm{X}$ betatron amplitude) with other distribution made small through simultaneous electron cooling and bunching, and (b) a longitudinally stretched beam created by cooling a coasting beam followed by bunching. Scans like Fig. 4 were made at four places using these two beam setups. The results are shown as + signs or circles (setups (a) and (b) respectively). The errors on the location of the best horizontal polarization are less than the size of the symbols. The longest polarization lifetimes were in excess of $1000 \mathrm{~s}$.

\section{6. ${ }^{3} \mathrm{He}$ beams}

The features of deuteron polarimetry described here apply as well to beams of other light polarized ions. For protons, extensive surveys have been available for scattering from carbon. ${ }^{10-11}$ The largest forward angle analyzing power is fortuitously close to the energy of and EDM storage ring using electric fields and operating at a momentum of $p=0.7 \mathrm{GeV} / \mathrm{c}$.

For ${ }^{3} \mathrm{He}$ the data base is much more sparse. The only measurements are double scattering made at Osaka at $443 \mathrm{MeV} \cdot{ }^{12}$ Nevertheless, the forward angle analyzing power is seen to be large. Thus it is possible to consider forward angle elastic scattering from carbon as a basis for polarization measurements.

\section{References}

1. F.J.M. Farley et al., Phys. Rev. Lett. 93, 052001 (2004).

2. R. Maier, Nucl. Instrum. Methods Phys. Res. Sect. A 390, 1 (1997).

3. D. Albers et al. Eur. Phys. J. A 22, 125 (2004).

4. J. Bisplinghoff et al., Nucl. Instrum. Methods Phys. Res. Sect. A 329, 151 (1993).

5. N.P.M. Brantjes et al., Nucl. Instrum. Methods Phys. Res. Sect. A 66449 (2012).

6. G.G. Ohlsen and P.W. Keaton, Jr., Nucl. Instrum. Methods 109, 41 (1973).

7. S.I. Serednyakov et al., Phys. Lett. 66B, 102 (1977).

8. I.B. Vasserman et al., Phys. Lett. B 187, 172 (1987); ibid. 198, 302 (1987).

9. A.W. Chao et al., Handbook of Accelerator Physics and Engineering, $2^{\text {nd }}$ edn., (World Scientific, Singapore, 2013).

10. E. Aprile-Gaboni et al., Nucl. Instrum. Methods 215, 147 (1983).

11. M.W. McNaughton et al., Nucl. Instrum. Methods in Phys. Res. A241, 435 (1985).

12. J. Kamiya et al. Phys. Rev. C 67, 064612 (2003). 